\title{
Numerical Testing of Series by Calculations to many Places of Decimals
}

1. The first query $(Q 1)$ and the first four replies $(Q R$ 1, 2, 3, 4) all dealt with the uses of tables to many places of decimals. This note indicates yet another use for many-figure calculations.

New functions are continually being studied, and new expansions or approximations are constantly being derived for these, as well as for other better-known functions. Whenever a new development is derived, it is clear that it is desirable to test its accuracy by using it numerically whenever an alternative process of calculation is available. Such a test is particularly desirable when the new development results from complicated processes and involves complicated coefficients.

In the case of a newly-derived series there is need for the calculation to be to many decimals in order to ensure adequate dispersion' of the effects of the various terms, and especially so that there may be a fair amount of variation in dispersion for different values of the variable or variables occurring in the series. Thus, in testing a series of which, say, the first six terms are available, it is of ten convenient to have the ratio of successive terms of the order of 500 to 2000 , so that each term has about three clear digits before the effect of the next term begins; a calculation to about eighteen digits would then be needed for the six terms. Again, it is sometimes possible when testing a complicated series numerically to use even more figures so that the effect of still later terms, not obtained or easily obtainable analytically, may be studied and approximate numerical values derived for the coefficients.

2. All these points may be illustrated by a study of the new series, given by LeHMER $^{1}$ (p. 133) for the very well-known Bessel function $I_{n}(x)$. This important expansion, as given by Lehmer, contains a number of misprints and errors, which will now be located and removed, mainly by means of numerical checks. The writer is fortunate in having available the following results, obtained in collaboration with W. G. BICKLEY during the preparation of a second volume of Bessel functions by the Committee for the Calculation of Mathematical Tables of the B.A.A.S.

$\begin{array}{rcc}n & I_{n}(3 n / 4) & \ln I_{n}(3 n / 4) \\ 4 & 0.325705181937935 & -1.121762656670127 \\ 8 & 0.422966068203539 & -0.860463320166327 \\ 12 & 0.633124136266149 & -0.457088768219174 \\ 16 & 1.004869122426993 & +0.004857306590037 \\ 20 & 1.647015253501582 & +0.498964712537913 \\ 24 & 2.755047680256368 & +1.013434749263236\end{array}$

3. First, a comparison between Lehmer's value of $\psi_{3}\left(x^{2}\right)$ on p. 133 and the coefficient of $\nu^{-3}$ in Watson's formula ${ }^{2}(2)$ indicates a discrepancy in the sign of the coefficient 16. For this early term in the series the methods suggested by both writers are easy to apply and it turns out that Lehmer's relation should read

$$
5760 \psi_{3}\left(x^{2}\right)=375 x^{6}-3654 x^{4}+1512 x^{2}+16
$$


(The anomaly of this term +16 is curious; it is the only one to break the alternation of sign which holds elsewhere.) The corresponding term on p. 134 also needs correction:

$$
\text { for }-1044097 / 175781250 n^{3} \text { read }-36521 / 7031250 n^{3} \text {. }
$$

Secondly, the sign of the term in $n^{-7}$ is + on p. 134 and - on p. 135. As it is a late and small term ( $0.0^{15} 45844$ in Lehmer's illustration) the sign '-' will be 'adopted' and verification left to the numerical test.

4. The formula for $\ln I_{n}(3 n / 4)$ may now be used, so modified in two places from the form given on p. 134, for the values $n=8(4) 24$. The results obtained, using the series curtailed at the term in $n^{-8}$, are listed in col. $(A)$; the smallest term used, that in $n^{-8}$, is listed in column $(B)$. Calculations are to 15 working decimals, no more being available for comparison.

\begin{tabular}{|c|c|c|c|c|c|c|c|}
\hline$n$ & $A$ & $\stackrel{B}{B 0^{-15} \times}$ & $C=\underset{10^{-16} X}{\mathrm{BAAS}}-A$ & $D=\underset{10^{-16} X}{\operatorname{BAAS}}-A_{c}$ & $E=D \times n^{10}$ & $\Delta$ & $\boldsymbol{F}$ \\
\hline 4 & & & & 329238885 & 0.3452 & & 0.25 \\
\hline 8 & -0.860463321670947 & 6952010 & 1504620 & 1482320 & 1.5916 & & 1.58 \\
\hline 12 & -0.457088768288568 & 271257 & 69394 & 47094 & 2.9160 & & 2.91 \\
\hline 16 & +0.004857306563877 & 27156 & 26160 & 3860 & 4.244 & & 4.24 \\
\hline 20 & +0.498964712515069 & 4556 & 22844 & 544 & 5.57 & & 5.57 \\
\hline 24 & +1.013434749240827 & 1060 & 22409 & 109 & $6.9(1)$ & & 6.90 \\
\hline
\end{tabular}

In col. $(C)$ are given the differences between BAAS results listed above and those in col. $(A)$. The results in col. $(C)$ for large $n$ suggest strongly that the differences are tending to a non-zero limit, that is, that there is a (numerical) error in the constant term $-\frac{1}{2} \ln (5 \pi / 2)$; this turns out to be the case: on p. 135 ,

$$
\text { for - } 1.03051030888407840331 \text {, read -1.0305103088 } 6177761966 .
$$

If this term is corrected, the values in col. (D) remain as the differences between the BAAS results and values given by Lehmer's series, corrected, as far as the term in $n^{-8}$. These corrected values (not given) are denoted by $\left(A_{c}\right)$. The value for $n=4$ in col. $(D)$ was computed directly from the corrected series.

5. Provided that no early term is in error, it may be expected that these differences $(D)$ will be of the form

$$
L / n^{9}+M / n^{10}+N / n^{11}+\cdots
$$

in which later terms will be less and less important as $n$ increases. To estimate the first two coefficients $L$ and $M$ several methods have been tried; the most convenient for the present example seems to be multiplication by $n^{10}$ followed by formation of differences: col. $(E)$, with $\Delta$, results. As it was hoped, for high $n$ the first difference $\Delta$ seems to tend to a limit as the effect of higher terms $N / n^{11}$, etc. dies out; this limit is slightly greater than 1.33 , although more figures are needed for $n=20$ and $n=24$ to estimate it more closely. Take $L=1.33 / 4=0.3325$ ( 4 being the interval in $n$ ); then from 
the value for $n=20$, using $0.3325 n+M=5.57$, it follows that $M=-1.08$. These two coefficients lead to the approximate values in col. $(F)$; the effect of omitted terms is obvious for $n=4$, but is less than 1 per cent elsewhere with $8 \leq n \leq 24$. Extrapolation outside the limit $n=24$ may be a little unsafe; but the last two terms given below for $n=100$ are probably correct.

6 . The approximate linearity of the values in col. $(E)$ for large $n$ also indicates the absence of gross errors in earlier terms; the ' - ' sign of the term in $n^{-7}$ is confirmed, for instance. It is instructive to consider the values which would have resulted from a wrong sign for this term; assuming the error in the constant term to have been eliminated, the values in col. $\left(D^{\prime}\right)$ would have been found in place of those in col. $(D)$. Multiplication by $n^{10}$ would then have given col. $\left(E^{\prime}\right)$, obviously not linear.

\begin{tabular}{|c|c|c|c|c|c|}
\hline$n$ & $\underset{10^{-15} \times}{D^{\prime}}$ & $E^{\prime}=D^{\prime} \times n^{10}$ & $\Delta$ & $G$ & $H$ \\
\hline 4 & - 5266987883 & - 5.523 & & & \\
\hline 8 & 42238202 & 45.353 & 39.030 & 8.212 & $(8 / 4)^{\circ}=8$ \\
\hline 12 & 2511765 & -155.522 & -110.169 & 3.429 & $\begin{array}{l}(12 / 8)^{3}=3.375 \\
(16 / 12)^{3}\end{array}$ \\
\hline 16 & 337706 & - 371.312 & -356.63 & 1.960 & $(20 / 16)^{3}=1.953$ \\
\hline $\begin{array}{l}20 \\
24\end{array}$ & 71088 & $\begin{array}{l}-727.94 \\
-1260.6\end{array}$ & -532.7 & 1.732 & $(24 / 20)^{3}=1.728$ \\
\hline
\end{tabular}

To find in which term an error seems likely, col. $(G)$ gives the ratios of successive terms in col. $\left(E^{\prime}\right)$. These values are clearly approximate cubes of ratios of successive values of $n$, thus indicating the term in $n^{-7}$ of the original series as the almost certain source of error. If two or more errors of comparable magnitude had been present this would have been evident from a considerable variation of the power of $(n+4) / n$ represented by the values in col. $(G)$. In such cases there will be at least two errors to find and a general systematic search is indicated, starting at and near the term giving the power most frequently represented by col. $(G)$.

7. For $n=100$, the following table of terms, corrected and extended from that given by Lehmer on p. 135, results:

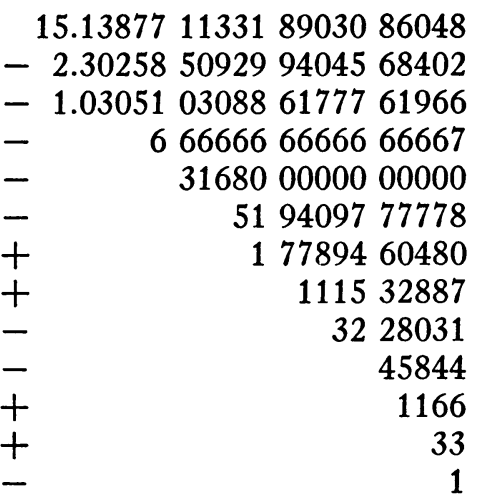

The sum is

11.80560589165142031925 
1 D. H. LEHMER, $M T A C$, p. 133-135.

${ }^{2}$ G. N. Watson, $A$ Treatise on the Theory of Bessel Functions, Cambridge, 1922, p. 228.

\section{RECENT MATHEMATICAL TABLES}

158[A].-H. S. UhLer, Exact Values of the first 200 Factorials, New Haven, Conn., privately published by H. S. Uhler, 1944,24 p. + cover. $16 \times 25.5$ cm. Photo-litho print. $\$ .80$

In $M T A C$, p. 163,125 , are records of unpublished tables of $n$ ! by JoFFE, $n \leqslant 100$, and by Salzer and Hillman, $n \leqslant 120$. Among previously printed tables the most extensive are those of Peters and Stein (J. T. Peters, Zehnstellige Logarithmentafel . . ., v. 1, 1922, Anhang, p. 58), $n \leqslant 60$; of F. RobBins, R. So. Edinburgh, Trans., v. 52, 1917, p. 167-174, $n \leqslant 50$; and of L. Potin, Formules et Tables Numériques . . ., Paris, 1925, p. 836, $n \leqslant 50$ (with four errors). The Introduction of the table under review, $n \leqslant 200$, (p. [iii-vi]) includes an account of "details of computation," "additional checks," "comparison with the results of others," "peculiarities of the table."

159[A].-Werner F. Vogel, Angular Spacing Tables, Detroit, Michigan, Vinco Corporation, 1943. iv, 233 p., hinged. $21.8 \times 28.5 \mathrm{~cm} . \$ 10.00$

Especially in connection with the spacing of teeth in such things as precision gears, splines, and index plates, are modern demands of a very high order. It is desirable to know within $0^{\prime \prime} .0005$ how many degrees, minutes and seconds are contained in angles between a line from the center of the gear through the center of one tooth to the line from the center of the gear through the center of any subsequent tooth on the gear. The main table of this volume (p. 1-208) provides such information for gears with 2(1)200 teeth. The presentation of the material is very clear. While seconds are given to $3 \mathrm{D}$, in every case where this decimal part is $\geqslant .5$ the entry appears as, for example, $35 .^{*} 510$, and the following footnote appears on each page: "*Star indicates that one second must be added when decimals are disregarded." For all practical purposes accuracy to the nearest 1 " suffices.

In the next three tables (p. 212-217), the values are given for $90^{\circ} / n, 180^{\circ} / n, 360^{\circ} / n$ where $n$ is the number of sides of a regular polygon, $n=4(1) 200$. In the first table the values of these angles are in degrees, minutes, and seconds to $4 \mathrm{D}$; in the second table they are given in degrees to $7 \mathrm{D}$; and in the third table in radians to $10 \mathrm{D}$. In each of these tables values corresponding to $n=1(1) 3$ are also given. On p. 218-227 is an 8-place table for converting any number of minutes and seconds up to $1^{\circ}$ into a decimal of a degree. The next table is for the conversion of $0\left(0^{\prime \prime} .001\right) 0^{\prime \prime} .999$ to decimals of a degree, $8 \mathrm{D}$. Under the heading "Important Constants," $\pi$ and $1 / \pi$ are given to $70 \mathrm{D} ; n \cdot \pi$ and $n \cdot(1 / \pi), n=1(1) 9$, to $35 \mathrm{D}$; $\pi / n$ and $1 /(n \pi), n=1(1) 19$, to $35 \mathrm{D}$; and $n \cdot \pi / 180$ and $n \cdot 180 / \pi, n=1(1) 9$, to 35D.

Mr. Vogel is now a member of the staff of Wayne University Engineering College in Detroit. As a former member of the Computing staff of J. T. Peters at the Astronomisches Recheninstitut of Berlin-Lichterfelde, we may be sure that he was most meticulous in every type of check to ensure the accuracy of his published tables.

R. C. A.

160[D, P].-General Electric Co., Trigonometric Functions of Half Center Angle of Regular Polygons (Standard Tables Division, Design Data, Mathematical Tables, Section G 902.4), February 22, 1944. 10 p. $20.5 \times 26.5 \mathrm{~cm}$. 